\title{
Examining Socioaffective Processing Biases in Cigarette Smokers with High Versus Low Trait Hostility
}

\author{
Adam M. Leventhal \\ Keck School of Medicine, University of Southern California \\ Christopher W. Kahler \\ Brown University
}

\begin{abstract}
Developing measures of socioaffective processing is important for understanding the mechanisms underlying emotional-interpersonal traits relevant to health, such as hostility. In this study, cigarette smokers with low $(\mathrm{LH} ; n=49)$ and high $(\mathrm{HH} ; n=43)$ trait hostility completed the Emotional Interference Gender Identification Task (EIGIT), a newly developed behavioral measure of socioaffective processing biases toward facial affect. The EIGIT involves quickly categorizing the gender of facial pictures that vary in affective valence (angry, happy, neutral, sad). Results showed that participants were slower and less accurate in categorizing the gender of angry faces in comparison to happy, neutral, and sad faces (which did not differ), signifying interference indicative of a socioaffective processing bias toward angry faces. Compared to LH individuals, HH participants exhibited diminished biases toward angry faces on error-based (but not speed-based) measures of emotional interference, suggesting impaired socioaffective processing. The EIGIT may be useful for future research on the role of socioaffective processing in traits linked with poor health.
\end{abstract}

Keywords: Emotional Interference Gender Identification Task, facial expressions, hostility, indirect measures, socioaffective processing

Affective processing bias-a tendency to automatically allocate attention preferentially toward certain classes of emotionally salient stimuli-may play a central role in the development and maintenance of various maladaptive traits. ${ }^{1} \mathrm{Cog}-$ nitive measures can be used to indirectly assess attentional responses toward emotional stimuli to make inferences regarding affective processing biases. The most common indirect measure of affective processing is the emotional Stroop task. $^{2}$ Consistent with the notion that individuals have implicit processing biases toward threat, evidence suggests that individuals are slower and less accurate in naming the color of threatening words, as compared to neutral words, on this task. ${ }^{3,4}$ This phenomenon, labeled an emotional interference

Correspondence should be addressed to Adam M. Leventhal, Ph.D., Institute for Health Promotion and Disease Prevention Research, Department of Preventive Medicine, Keck School of Medicine, University of Southern California, 1000 South Fremont Ave., Unit 8, Alhambra, CA 91803, USA.E-mail: adam.leventhal@usc.edu effect, may occur because an individual's attention is distracted by the affective content of threatening stimuli, which disrupts color naming performance. Importantly, extant data indicate that emotional interference effects are abnormally elevated or diminished among individuals with maladaptive emotional traits ${ }^{2}$ and tobacco dependence. ${ }^{5}$ Thus, impaired affective processing may contribute to characteristics linked with poor mental and physical health.

Despite the clinical and scientific promise of the emotional Stroop task, it is not without its limitations. Of note, participants can use conscious strategies, such as visual defocusing, while completing the task, ${ }^{2,6}$ which can serve to suppress emotional interference effects. Thus, researchers have been testing new tasks designed to assess emotional interference effects induced by socioaffective stimuli (i.e., emotional faces), which may be less vulnerable to this pitfall. For example, Kolassa and Miltner ${ }^{7}$ compared people with social phobia $(n=19)$, spider phobia $(n=19)$, and healthy controls $(n=19)$ on a speeded gender identification task. In this 
task, participants categorized the gender of pictures of human faces expressing angry, happy, or neutral emotion. Because participants have to focus on the target stimulus to identify its gender, this task may be less vulnerable to some of the strategies used to suppress emotional interference effects. The authors found that participants were slower to identify the gender of angry and happy faces as compared to neutral faces, indicative of a socioaffective processing bias towards these emotions. No differences in affective biases were found between the groups.

Based on these findings, there are several issues that require further clarification. First, angry expressions were the only negatively valenced stimuli that were used, leaving it unclear whether socioaffective interference effects extend to other negative affects (e.g., sadness). Second, reaction times were the only behavioral outcome analyzed. Error rate can also be an additional useful indicator of emotional interference. The capture of attention by the emotional content of stimuli may not only interfere with the speed of the required response on an emotional interference task, it can also interfere with accuracy of that response. Indeed, research using the emotional Stroop task indicates that error rate can be an additional useful indicator of emotional interference because it associates with relevant external variables, but it may potentially tap a unique component of affective processing biases. ${ }^{3,8-10}$ For example, Dawkins et al ${ }^{10}$ found that administering nicotine to participants resulted in increased levels of interference by positive (vs neutral) words on an emotional Stroop task when error rates were used as the outcome variable but did not find significant effects when speed was used as the outcome variable. Similarly, Waters et $\mathrm{al}^{9}$ found a similar nicotine (vs placebo) effect with error rates (but not response latency) on interference induced by smoking-related words. Given their relationship with relevant external variables, error rate measures of emotional interference appear to be valid indicators of affective processing, which underscores the significance of measuring both reaction time and error rate in the assessment of socioaffective processing. Finally, it would be informative to examine whether emotional interference effects on the gender identification task differ as a function of other maladaptive traits that are relevant to mental and physical health.

Hostility is one such trait that might be associated with dysregulated socioaffective face processing. This trait is defined by the presence of cynical attitudes, mistrust of others, irritability, and a tendency toward anger expression. ${ }^{11}$ Of note, hostility is a robust risk factor for cardiovascular diseases $^{12}$ and negative health behaviors, including alcohol consumption, ${ }^{12}$ smoking, ${ }^{12}$ and poor smoking cessation outcomes. ${ }^{13}$ As a result, there has been increased interest in examining the psychological processes that underlie hostility. Studies of college students have found that higher levels of hostility are associated with a tendency to be less accurate in decoding facial expressions of emotion. ${ }^{14,15}$ However, it remains unclear whether hostility is also related to abnormal- ities in socioaffective face processing on indirect measures that may tap implicit emotional interference effects. In addition, it is unknown whether hostility is linked with socioaffective processing biases in populations at greater risk of negative health outcomes, such as smokers, which is of interest because of the high prevalence of smokers among hostile individuals. Finally, it is not clear whether associations between hostility and dysregulated socioaffective processing may be due to hostility's overlap with general emotional disturbance, or whether they are specific to the socioaffective construct of hostility.

In this study, individuals with low hostility ( $\mathrm{LH})$ and high hostility $(\mathrm{HH})$ completed an Emotional Interference Gender Identification Task (EIGIT), in which participants made speeded categorizations of the gender of facial pictures that varied in affective valence. The participants were smokers enrolled in a more extensive laboratory study on hostility and smoking ${ }^{36}$ who completed the EIGIT at their baseline visit. The primary aims of this study were to: (1) investigate socioaffective processing biases by comparing speed and accuracy of responses to angry, happy, neutral, and sad faces on the EIGIT; and (2) examine whether these biases were moderated by trait hostility.

\section{METHODS}

\section{Participants}

Participants were LH $(n=49)$ and HH $(n=43)$ smokers recruited from the local community to participate in a more extensive laboratory study on hostility and smoking. ${ }^{36}$ Participants were recruited through advertisements in Rhode Island area periodicals, with announcements targeting smokers aged 18 years and older interested in participating in research on emotion and smoking. For inclusion, participants had to (a) be 18 years of age or older, $(b)$ have smoked cigarettes regularly for at least 1 year, $(c)$ currently smoke at least 10 cigarettes per day, $(d)$ currently be using no other tobacco products or nicotine replacement therapy, and $(e)$ be able to read English. Subjects were excluded if they were dependent on alcohol or drugs other than nicotine and met criteria for current affective disorder as determined by the Structured Clinical Interview for DSM-IV-Non Patient Version (SCIDNP). ${ }^{16}$

The overall sample was $54.3 \%$ female, $73.9 \%$ white, and had a mean age of 38.7 years $(S D=12.5$, range $=18-68)$. Twelve percent of the sample reported currently taking psychiatric medications, and $42 \%$ met criteria for history of major depressive disorder. On average, participants smoked $21.5(S D=8.7)$ cigarettes per day and had a Fagerström Test of Nicotine Dependence (FTND) ${ }^{17}$ score of $5.2(S D=2.2)$, indicating a medium level of nicotine dependence severity in this sample. None of the aforementioned baseline variables significantly differed by group. On the Positive and Negative 
Affect Schedule (PANAS) ${ }^{18} \mathrm{HH}$ participants scored higher than LH participants on the negative affect scale (LH, $M=$ $12.0, S D=2.7$; HH, $M=15.4, S D=6.2 ; p=.0007)$ but not on the positive affect scale (LH, $M=33.4, S D=9.1$; $\mathrm{HH}, M=31.9, S D=9.2 ; p=.44)$. All participants provided informed consent before participating, and the study's procedures were approved by the university's Institutional Review Board.

\section{Self-Report Measures}

\section{The 17-Item Cook-Medley Hostility Scale}

The 17-Item Cook-Medley Hostility Scale ${ }^{11}$ is an empirically-derived, shortened version of the 50-item truefalse Cook-Medley Hostility Scale drawn from the Minnesota Multiphasic Personality Inventory (MMPI). ${ }^{19}$ This shortened scale correlates strongly with the extended version $(r>.93)$ and taps a primary latent dimension indicative of expectations of a hostile and oppositional interpersonal world, ${ }^{11}$ which may be more relevant than broader hostility constructs to socioaffective processing. Subjects had to score either a 5 or lower $(\mathrm{LH})$ or a 10 or higher $(\mathrm{HH})$ to participate. Using these cut points ensured substantial differentiation between groups as they corresponded with the upper $(\mathrm{HH}$; score of $\geq 10)$ and lower $(\mathrm{LH} ; \leq 5)$ thirds of scores from previous samples. ${ }^{20}$ This was expected to maximize the differentiation between the two groups on the target construct, which we anticipated would increase the power of the hostility group contrast. To avoid confounds, $\mathrm{LH}$ and $\mathrm{HH}$ groups were recruited to ensure an approximate balance of gender and Fagerström Test for Nicotine Dependence score.

\section{Positive and Negative Affect Schedule}

The PANAS ${ }^{18}$ includes two 10 -item subscales that measure current positive (e.g., interested, excited, enthusiastic) and negative (e.g., irritable, distressed, nervous) affect. This measure was included as a potential covariate to examine whether effects involving hostility were above and beyond any overlap with general emotional disturbance.

\section{Procedure}

During a phone screen, participants were administered the 17-item Cook-Medley Hostility Scale and asked questions about eligibility criteria. Eligible individuals were invited for a baseline session. At the outset of the baseline session, participants were administered the SCID-NP. They then completed questionnaires, including the PANAS, and then responded to the EIGIT.

\section{The EIGIT}

The EIGIT was programmed using E-prime software (Version 1.1$)^{21}$ and administered on a Pentium-IV computer. Instructions were presented on a 19-inch monitor, and all responses were recorded by the computer. Responses were entered directly on the computer's keyboard.

\section{Task Procedure}

At the outset of the task, participants were instructed that the task examined detection speed and accuracy. They were informed that they would be presented with pictures of faces on the computer screen, and that their task was to identify the gender of each face by pressing a corresponding button on the keyboard as quickly and as accurately as possible. Key assignment (left-male/right-female vs leftfemale/right-male) was randomized across participants. Participants first completed 8 practice trials; after each, corrective feedback was presented (correct or incorrect). They then completed 32 experimental trials, for which feedback was not provided.

On each trial, a target stimulus was presented and remained on the screen until the participant responded. An interstimulus interval ranging from 1000 to $1500 \mathrm{~ms}$ elapsed between each trial. The duration of this interval was random across trials to prevent participants from guessing the time at which the target would appear.

\section{Stimuli}

Pictures were selected from the Japanese and Caucasian Facial Expressions of Emotion system (JACFEE-NEUF). ${ }^{22}$ The JACFEE-NEUF includes pictures of posers who were photographed while portraying specific emotions. Posers were trained to contract or relax different facial muscles associated with the specific emotions. The background and lighting of pictures are consistent across the stimulus set. For the development of JACFEE-NEUF, photos were coded by Ekman and Friesen's ${ }^{23}$ Facial Action Coding System to ensure the validity of the expressions to portray the intended emotion, as well as comparability within emotion of expression intensity levels. ${ }^{22}$ Pictures were 4.5 inches wide by 3.25 inches tall and presented at the center of the screen. There were 8 ( 4 female, 4 male) practice trials using neutral pictures, followed by 32 experimental trials: 8 angry ( 4 female, 4 male), 8 happy ( 4 female, 4 male), 8 neutral ( 4 female, 4 male), and 8 sad ( 4 female, 4 male). The stimuli selected from the JACFEE -NEUF system were as follows: practice (N7, N10, N18, N23, N37, N42, N45, N53), angry (E1-E8), happy (E33-E40), neutral (N1, N4, N19, N20, N30, N33, N46, N48), and sad (E41-E48). Within each of the affective categories, there were 2 photographs of Caucasian women, 2 of Caucasian men, 2 of Japanese women, and 2 of Japanese men. Each stimulus presented a photograph of a different person to avoid the development of associations between particular posers and affective valences, which could contaminate responses across categories (e.g., seeing a particular poser express an angry face on 1 trial could later bias an individual's response to a picture of the same poser expressing a neutral face). To minimize the 
influence of factors other than emotional valence on the speed and accuracy of gender identification, pictures were selected such that the degree to which male posers appeared masculine and female posers appeared feminine was consistent across emotion categories. The order of presentation was random.

\section{Scoring}

Consistent with prior reports, ${ }^{24,25}$ reaction times (RTs) were discarded for trials with incorrect responses $(3.8 \%$ of trials) and responses that were less than $300 \mathrm{~ms}$ or more than 3 SDs of each participant's mean RT ( $2.0 \%$ of trials) to remove outliers. The remaining trials were used to calculate each participant's mean RT for the 8 trials within each emotion category. Error rates were computed for each participant by dividing the number of incorrect responses by the number of trials (i.e., 8) within each category. These 2 indices served as the primary dependent variables.

Slower RTs and more errors on trials of an emotional category versus neutral trials are indicative of a socioaffective processing bias toward that emotional expression. Thus, for the purpose of evaluating the psychometric properties of EIGIT biases, separate interference effect difference scores were computed for angry, happy, and sad categories (mean of emotionally valenced trials minus the mean of neutral trials). The intercorrelations of interference effects for RT and error estimates are reported in Table 1. Following Waters et al, ${ }^{9}$ we estimated internal reliability by randomly separating 2 groups of 4 stimuli within each category, computing their means, and then subtracting the mean value of the neutral trials to create 2 parallel interference effect difference scores for each stimulus category. We then correlated these difference scores and applied the Spearman-Brown formula to derive the split-half reliability coefficient (see values on diagonal in Table 1).

TABLE 1

Internal Reliability and Intercorrelations of EIGIT Interference Effects

\begin{tabular}{lrrrrrr}
\hline & 1. & 2. & 3. & 4. & 5. & 6. \\
\hline 1. Angry (RT) & $(.53)$ & & & & & \\
2. Happy (RT) & $.46^{*}$ & $(.43)$ & & & & \\
3. Sad (RT) & $.46^{*}$ & $.61^{*}$ & $(.55)$ & & & \\
4. Angry (error rate) & $-.28^{*}$ & $-.26^{*}$ & -.15 & $(.24)^{\mathrm{a}}$ & & \\
5. Happy (error rate) & $-.23^{*}$ & -.13 & -.17 & $.37^{* \mathrm{a}}$ & $(.38)^{\mathrm{a}}$ & \\
6. Sad (error rate) & -.17 & -.14 & -.18 & $.41^{* \mathrm{a}}$ & $.59^{* \mathrm{a}}$ & $(.72)^{\mathrm{a}}$ \\
\hline
\end{tabular}

Notes. $N=92$. Analyses use interference effect difference scores (mean of emotionally-valenced trials - mean of neutral trials). Values on the diagonal represent split-half reliability coefficients. RT $=$ Reaction Time. a Spearman's $\rho$ presented because error rates were not normally distributed.

${ }^{*} p<.05$.

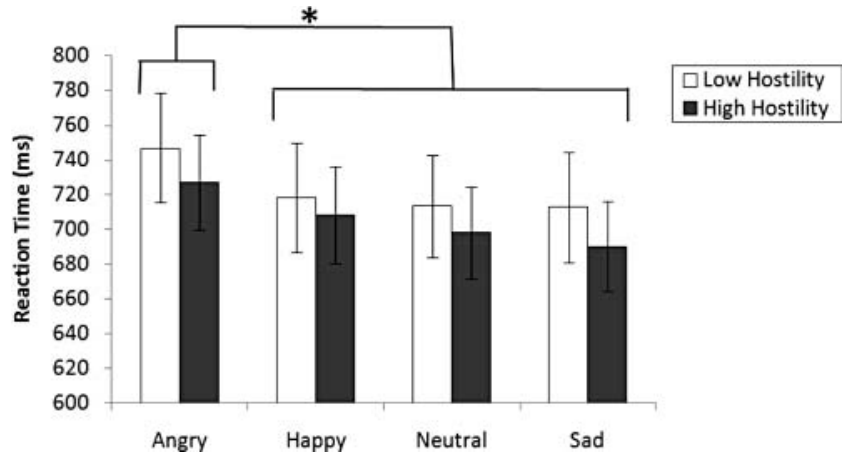

FIGURE 1 Means and standard errors of reaction times (RTs) for angry, happy, neutral, and sad stimuli, by group. Notes. There were significant differences in RTs for angry faces as compared to happy, neutral, and sad faces. ${ }^{*} p \mathrm{~s}<.05$.

\section{Design and Data Interpretation}

This study uses a mixed 2-way factorial design with the between-subjects factor of Group (LH vs $\mathrm{HH}$ ) crossed with the within-subjects factor of Emotion (angry vs happy vs neutral vs sad stimuli on the EIGIT). An Emotion main effect would indicate a socioaffective processing bias, whereby participants demonstrate greater interference by 1 emotional valence versus another. A Group $\times$ Emotion interaction effect would indicate that socioaffective processing biases differed as a function of hostility.

\section{RESULTS}

\section{Reaction Times}

A mixed Group (LH vs HH) $\times$ Emotion (angry vs happy vs neutral vs sad) ANOVA was performed for RTs, using the SAS GLM program for unbalanced cell sizes with the multivariate option to avoid violations of sphericity assumption. ${ }^{26}$ The ANOVA yielded a significant main effect of Emotion, $F(3,88)=4.04, p=.0097, \eta^{2}=.12$, indicative of a socioaffective processing bias in the overall sample. The main effects of Group and the interaction were not significant. Follow-up tests indicated that RTs for angry trials were significantly slower than for happy $(p=.049)$, neutral $(p=.005)$, and sad $(p=.001)$ trials. There were no significant differences in RTs between happy, neutral, and sad trials (see figure 1).

\section{Error Rates}

Because errors rates were not normally distributed, nonparametric tests were used. Wilcoxin sign-rank tests of differences in error rates as a function of Emotion type indicated that participants made significantly more errors on angry trials than on happy, neutral, or sad trials $(S s \geq 230$, ps $\leq .0002)$. Nonparametric rank from 2-way tests 27 indicated a significant omnibus Group $\times$ Emotion interaction, 


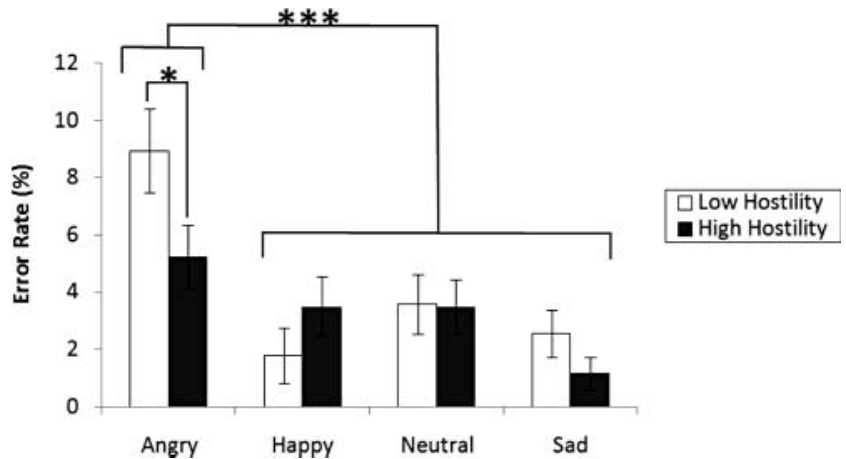

FIGURE 2 Means and standard errors of error rates for angry, happy, neutral, and sad stimuli, by group. Notes. There were significant differences in error rates for angry faces as compared to happy, neutral, and sad faces $\left({ }^{* * *} p \mathrm{~s} \leq .0006\right)$. There was also was a significant Group X Emotion interaction, such that there were trend differences between $\mathrm{HH}$ and LH participants in error rates for angry faces $\left({ }^{*} p=.052\right)$, but no group differences in error rates for other emotion categories ( $p \mathrm{~s} \geq .17$ ).

$F(3,270)=3.10, p=.03$. To deconstruct the interaction, Group differences were tested for each emotion category. Mantel-Haenszel $\chi^{2}$ analyses demonstrated a trend indicating that a greater proportion of LH participants made more errors than $\mathrm{HH}$ participants for angry trials, Mantel-Haenszel $\chi^{2}(1)=3.76, p=.053$ (see Figure 2). In the LH group, $51 \%$ made 0 out of 8 errors, $27 \%$ made 1 error, and $22 \%$ made 2 errors. In the $\mathrm{HH}$ group, $63 \%$ made 0 errors, $33 \%$ made 1 error, and $5 \%$ made 2 errors. There were no significant group differences in the proportion of errors for happy, neutral, and sad trials. Additional nonparametric rank tests, controlling for PANAS-negative affect scores, continued to show a significant Group $\times$ Emotion interaction, $F(3,267)=3.14, p=.03$

\section{COMMENT}

This study examined the responses of $\mathrm{HH}$ and LH individuals on an EIGIT, which assessed the relative emotional interference of angry, happy, neutral, and sad facial expressions. Results showed that participants were slower and less accurate in responding to angry faces in comparison to happy, neutral, and sad faces (which did not differ from one another). This suggests that participants were distracted by the affective content of angry faces, which interfered with their ability to quickly and accurately categorize the gender of these faces. The greater interference caused by angry faces is consistent with Kolassa and Miltner, ${ }^{7}$ who found that participants had slower responses on the EIGIT to angry faces, as compared to neutral faces. The current study extends these findings by demonstrating that the EIGIT is sensitive to emotional interference effects as a result of angry expressions for both RT and accuracy.

Although Kolassa and Miltner ${ }^{7}$ found that participants exhibited slower RTs on happy (vs neutral) expressions on an
EIGIT, the accuracy and speed of responses were not different on happy versus neutral trials in this study. It is possible that differences in sample characteristics and task procedures could explain this discrepancy. Two-thirds of the participants in Kolassa and Miltner had an anxiety disorder. In addition, their study included more trials and half of the participants had completed an emotional facial expression-decoding task prior to the EIGIT. These differences are of note, given that Bradley et $\mathrm{al}^{28}$ found that socioaffective processing biases toward happy faces on a visual probe task (i.e., a test of visuospatial attention toward emotional faces) were exhibited only in anxious patients during the latter half of trials. Thus, socioaffective processing biases toward happy affect may be more easily detectible in the latter half of trials and among anxious individuals. Nevertheless, other studies using the visual probe task have not demonstrated a significant processing bias toward happy faces. ${ }^{29,30}$

Participants were not slower or less accurate in responding to sad faces. Previous studies have had mixed findings, with several showing no evidence of a sad face-processing bias. ${ }^{31}$ Typically, studies that have found significant biases toward sad faces demonstrate such effects only in depressed participants. ${ }^{32,33}$ Thus, the overall pattern of data across studies suggests that nondepressed participants do not show processing biases toward sad faces.

Examination of whether trait hostility moderated socioaffective processing biases indicated that $\mathrm{HH}$ and $\mathrm{LH}$ participants exhibited different patterns of errors across the emotional categories (i.e., a significant Group $\times$ Emotion interaction). This interaction remained significant when controlling for PANAS-negative affect scores, indicating that this effect was not explained by higher overall levels of negative affectivity in $\mathrm{HH}$ individuals; rather, it was specific to the socioaffective trait of hostility. Simple effect analyses indicated a statistical trend $(p=.052)$, such that LH individuals tended to be less accurate than $\mathrm{HH}$ individuals in categorizing the gender of angry faces; however, there were no group differences in participants' responses to the other emotional categories. These findings suggest that HH individuals exhibited less pronounced socioaffective processing biases toward angry expressions than did LH individuals, although the magnitude of this effect was modest. It is possible that because $\mathrm{HH}$ people experience high levels of interpersonal cynicism and anger in their daily life, they have become habituated to hostile interpersonal stimuli, such as angry faces. Thus, they may be less surprised and distracted by angry faces, which would explain the lower error rates in this group. By contrast, LH individuals who experience low levels of social cynicism may be more sensitive to hostile interpersonal stimuli, and thus more surprised and distracted by angry faces. One potential interpretation is that diminished interference effects among $\mathrm{HH}$ individuals represent impaired socioaffective processing. Indeed, previous studies have found that scores on the Cook-Medley Hostility Scale and other measures of hostility that tap social cynicism are 
associated with lower detection ability on direct measures of facial affect decoding. ${ }^{14,15}$ Taken together, hostility may potentially be linked with processing deficits in differentiating the emotional salience of facial expressions on both direct and indirect measures.

Hostility did not moderate emotional interference effects when RT was the dependent variable. In addition, correlations between RT- and error-based estimates of emotional interference on the EIGIT were small in magnitude and negative in direction. Executing the required response in the EIGIT (i.e., gender classification) accurately is quite simple, and errors were relatively infrequent in this study. By contrast, executing this response quickly can be relatively difficult, and there was a range of RTs both above and below the mean response latency. Thus, errors on the EIGIT may represent particularly severe capture of attention by the emotional content of stimuli, resulting in significant disruption of the required response, whereas emotion-induced response slowing may be more sensitive to subtle attentional interferences. This outcome could perhaps explain the disparity in findings across error and RT. Indeed, previous studies using the emotional Stroop task have found that error rates and RTs have differential associations with external variables, and that these measures do not consistently correlate with each other. ${ }^{9,10}$ These dissociations in the present study and previous research may indicate that RT and error measures capture different components of emotional interference and highlight the importance of considering both indices when using the EIGIT.

This study had several limitations. First, this study did not fully clarify the psychometric properties of the EIGIT. This study used a brief version of the EIGIT with relatively few trials. Although the split half-reliability estimates of interference effects were comparable to previous estimates reported in emotional Stroop studies that used a larger number of trials, ${ }^{9}$ these values were generally modest in size. The pattern of intercorrelations indicated that within RT and error rate indices, interference effects for emotion categories were associated with one another. Although there have been reports that interference scores are correlated across stimulus categories in emotional Stroop studies, ${ }^{34}$ it is unclear what this means with regards to the validity of the EIGIT. Second, the findings were specific to angry expressions. Although the lack of corresponding effects with sad faces indicates that the findings were particular to social signals of threat (and not simply general negative affect), it is unclear whether this pattern would be illustrated with fearful facial expressions, which also signal impending threat. Third, we did not control for type-I error, although several analyses were conducted. In addition, overall error rates in EIGIT performance were low; thus, their practical meaning is not entirely clear. Further, even though a significant Group X Emotion type interaction was found for error rate, simple effect analyses indicated that $\mathrm{LH}$ and $\mathrm{HH}$ differences in responding to angry faces were only at a trend level $(p=.052)$. Accordingly, these results should be interpreted with caution. On a related note, the sample size may have been too small to detect some of the effects present herein. Indeed, given the size of the withinparticipant emotion effect contrast of RT on happy versus neutral faces, the post-hoc calculated power for detecting that the effect was only 0.14 , which is insufficient. Perhaps using a blocked (instead of mixed) format for presenting each emotion type, which has been shown to produce more robust effects in Stroop studies, ${ }^{35}$ and examining these effects in larger samples would allow a more sensitive test of the current hypotheses. Fourth, we did not analyze whether the gender of the participant and the target stimuli moderated interference effects because of limited sample size and the small number of trials in this task. Nonetheless, this issue should be addressed in future work. Fifth, the generalizability of the current findings is not completely clear. Because the present study was focused on smokers with relatively high rates of past major depression who score on the lower and upper ends of the hostility continuum, it is unclear whether the current findings would extend to nonsmokers and individuals with moderate levels of hostility. Although prior research with the EIGIT suggests that emotional interference effects are found in nonsmokers who are not recruited with regards to level of hostility ${ }^{7}$ and data using other interference tasks have extended to a range of clinical and nonclinical populations, ${ }^{1-10}$ it will be important for future research to explore whether some of the findings that were particular to the current study will generalize to these other groups. Finally, although the EIGIT is suspected to measure similar processes to the emotional Stroop task and be less prone to strategic efforts to suppress interference effects, we cannot empirically test these hypotheses. Thus, future studies should explore the relation between the EIGIT and other measures of affective processing.

In sum, this study showed that the EIGIT is sensitive to socioaffective processing biases toward angry faces and offered preliminary evidence that error-based estimates of these biases are moderated by trait hostility. Because the EIGIT uses ecologically valid affective stimuli and may be less prone to strategic efforts to suppress interference effects, this task may be useful for future studies examining the role of socioaffective processing biases in emotional and interpersonal traits that contribute to mental and physical health.

\section{ACKNOWLEDGEMENTS}

This study was supported by grant R21 DA019628 from the National Institute on Drug Abuse to Christopher Kahler and grant K08 DA025041 from the National Institute on Drug Abuse to Adam Leventhal. The authors gratefully acknowledge Jennifer Larence, Dan Belenky, Catherine Costantino, Cheryl Eaton, and Timothy Souza for their assistance on this project. 


\section{REFERENCES}

[1] Macleod C. The Stroop task in clinical research. In: Wenzel A, Rubin D, eds. Cognitive Methods and Their Application to Clinical Research. Washington DC: American Psychological Association; 2005:41-2.

[2] Williams JM, Mathews A, MacLeod C. The emotional Stroop task and psychopathology. Psychol Bull. 1996;120:3-24.

[3] Eschenbeck H, Kohlmann C, Heim-Dreger U, Koller D, Lesser M. Processing bias and anxiety in primary school children: a modified emotional Stroop colour-naming task using pictoral facial expressions. Psychol Sci. 2005;46:451-465.

[4] McKenna F, Sharma D. Intrusive cognitions: an investigation of the emotional Stroop task. J Exp Psychol. 1995;21:1595-1607.

[5] Powell J, Tait S, Lessiter J. Cigarette smoking and attention to signals of reward and threat in the Stroop paradigm. Addiction. 2002;97:1163-1170.

[6] Amir N, Freshman M, Foa E. Enhanced Stroop interference for threat in social phobia. J Anx Disorders. 2002;16:1-9.

[7] Kolassa IT, Miltner WH. Psychophysiological correlates of face processing in social phobia. Brain Res. 2006;1118:130-141.

[8] Duka T, Townshend JM, Collier K, Stephens DN. Kindling of withdrawal: a study of craving and anxiety after multiple detoxifications in alcoholic inpatients. Alcohol Clin Exp Res. 2002;26:785-795.

[9] Waters AJ, Shiffman S, Sayette MA, Paty JA, Gwaltney CJ, Balabanis $\mathrm{MH}$. Attentional bias predicts outcome in smoking cessation. Health Psychol. 2003;22:378-387.

[10] Dawkins L, Powell JH, West R, Powell J, Pickering A. A double-blind placebo controlled experimental study of nicotine: I-effects on incentive motivation. Psychopharmacology (Berl). 2006;189:355-367.

[11] Strong DR, Kahler CW, Greene RL, Schinka J. Isolating a primary dimension within the Cook-Medley hostility scale: a Rasch analysis. Pers Individ Dif. 2005;39:21-33.

[12] Miller TQ, Smith TW, Turner CW, Guijarro ML, Hallet AJ. A metaanalytic review of research on hostility and physical health. Psychol Bull. 1996;119:322-348.

[13] Kahler CW, Spillane NS, Leventhal AM, Strong DR, Brown RA, Monti PM. Hostility and smoking cessation treatment outcome in heavy social drinkers. Psychol Addict Behav. 2009;23:67-76.

[14] Larkin KT, Martin RR, McClain SE. Cynical hostility and the accuracy of decoding facial expressions of emotions. J Behav Med. 2002;25:285-292.

[15] Knyazev G, Bocharov A, Slobodskaya H, Ryabichenko T. Personalitylinked biases in perception of emotional facial expression. Pers Individ Dif. 2008;44:1093-1104.

[16] First MB, Spitzer RL, Gibbon M, Williams JBW. Structured Clinical Interview for DSM-IV-TR Axis I Disorders, Research Version, NonPatient Edition (SCID-I/NP). New York: Biometrics Research, New York State Psychiatric Institute; 2002.

[17] Heatherton TF, Kozlowski LT, Frecker RC, Fagerström K-O. The Fagerström Test for Nicotine Dependence: a revision of the Fagerström Tolerance Questionnaire. Brit J Addict. 1991;86:1119-1127.
[18] Watson D, Clark LA, Tellegen A. Development and validation of brief measures of positive and negative affect: the PANAS scales. J Pers Soc Psychol. 1988;54:1063-1070.

[19] Cook W, Medley D. Proposed hostility and pharasaic-virtue scales for the MMPI. J Appl Psychol. 1954;38:414-418.

[20] Han K, Weed NC, Calhoun RF, Butcher JN. Psychometric characteristics of the MMPI-2 Cook-Medley Hostility scale. J Pers Assess. 1995;65:567-585.

[21] Schneider W, Eschman A, Zuccolotto A. E-Prime User's Guide. Pittsburgh, PA: Psychology Software Tools Inc; 2002.

[22] Matsumoto D, Ekman P. Japanese and Caucasian Facial Expressions of Emotion (JACFEE) and Neutral Faces (JACNeuF). Berkeley, CA: Paul Ekman; 2004.

[23] Ekman P, Friesen W. Facial Action Coding System: A Technique for the Measurement of Facial Movement. Palo Alto, CA: Consulting Psychologists Press; 1978.

[24] Kolassa IT, Kolassa S, Musia F, Miltner W. Event-related potential to schematic faces in social phobia. Cogn Emot. 2007;21:1721-1744.

[25] Putman P, Hermans EJ, Koppeschaar H, van Schijndel A, van Honk J. A single administration of cortisol acutely reduces preconscious attention for fear in anxious young men. Psychoneuroendocrinology. 2007;32:793-802.

[26] The SAS System for Windows [computer program]. Version 8.2. Cary, NC: SAS Institute Inc; 2003.

[27] Toothaker AK, Newman D. Nonparametric competitors to the twoway ANOVA. J Educ Behav Stat. 1994;19:237-273

[28] Bradley BP, Mogg K, White J, Groom C, de Bono J. Attentional bias for emotional faces in generalized anxiety disorder. Brit J Clin Psychol. 1999;38:267-278.

[29] Bradley BP, Mogg K, Millar N, et al. Attentional biases for emotional faces. Cogn Emot. 1997;11:25-42.

[30] Mogg K, Millar N, Bradley BP. Biases in eye movements to threatening facial expressions in generalized anxiety disorder and depressive disorder. J Abnorm Psychol. 2000;109:695-704.

[31] Bradley BP, Mogg K, Millar N. Covert and overt orienting of attention to emotional faces in anxiety. Cogn Emot. 2000;14:789-808.

[32] Gotlib IH, Kasch KL, Traill S, Joormann J, Arnow BA, Johnson SL. Coherence and specificity of information-processing biases in depression and social phobia. J Abnorm Psychol. 2004;113: 386-398.

[33] Joormann J, Gotlib IH. Selective attention to emotional faces following recovery from depression. J Abnorm Psychol. 2007;116:80-85.

[34] Drobes DJ, Elibero A, Evans DE. Attentional bias for smoking and affective stimuli: a Stroop task study. Psychol Addict Behav. 2006;20:490-495.

[35] Waters AJ, Sayette MA, Franken IH, Schwartz JE. Generalizability of carry-over effects in the emotional Stroop task. Behav Res Ther. 2005;43:715-732.

[36] Kahler CW, Leventhal AM, Colby SM, Gwaltney CJ, Kamarck TW, Monti PM. Hostility, cigarette smoking, and responses to a lab-based social stressor. Exp Clin Psychopharmacol. 2009;17:413-424. 
Reproduced with permission of the copyright owner. Further reproduction prohibited without permission. 